\title{
Self-stabilizing temperature-driven crossover between topological and nontopological ordered phases in one-dimensional conductors
}

\author{
Bernd Braunecker ${ }^{1}$ and Pascal Simon ${ }^{2}$ \\ ${ }^{1}$ SUPA, School of Physics and Astronomy, University of St. Andrews, North Haugh, St. Andrews KY16 9SS, United Kingdom \\ ${ }^{2}$ Laboratoire de Physique des Solides, CNRS UMR-8502, Université de Paris Sud, 91405 Orsay Cedex, France
}

(Received 22 October 2015; published 28 December 2015)

\begin{abstract}
We present a self-consistent analysis of the topological superconductivity arising from the interaction between self-ordered localized magnetic moments and electrons in one-dimensional conductors in contact with a superconductor. We show that, due to a gain in entropy, there exists a magnetically ordered yet nontopological phase at finite temperatures that is relevant for systems of magnetic adatom chains on a superconductor. The spin-orbit interaction is taken into account, and we show that it causes a modification of the magnetic order, yet without affecting the topological properties.
\end{abstract}

DOI: 10.1103/PhysRevB.92.241410

PACS number(s): 71.10.Pm, 74.20.Mn, 74.78.Na, 75.30.Hx

Introduction. Topological superconductors have received much attention recently, partly because they host exotic low energy excitations such as Majorana bound states [1-3], whose non-Abelian statistics are attractive for topological quantum computation $[4,5]$. As a remarkable feature, topological superconductivity can be created artificially by contacting specific materials with a conventional $s$-wave superconductor. For instance, it arises at the interface between the surface states of a three-dimensional topological insulator and an $s$-wave superconductor [6], in one-dimensional (1D) semiconducting wires with a strong spin-orbit interaction (SOI) and a Zeeman magnetic field with proximitized superconductivity [7-11], or in arrays of magnetic nanoparticles or magnetic adatoms on top of a superconducting surface [12-22], such as iron adatoms on lead [23-25].

The systems we consider in this Rapid Communication exhibit a topological phase emerging from self-organization of magnetic moments embedded in 1D conductors with proximity induced superconductivity. This situation may apply to semiconducting wires with extrinsic magnetic impurities or intrinsic moments such as nuclear spins, or a conducting wire made of magnetic adatoms on a superconducting surface. Due to the Ruderman-Kittel-Kasuya-Yosida (RKKY) interaction mediated through the electrons, the magnetic moments can undergo an ordering transition below a temperature $T^{*}$ and form a spiral with a spatial period characterized by the wave number $2 k_{m}$ (see Fig. 1 ) such that $k_{m}=k_{F}$, for $k_{F}$ the Fermi momentum. This ordering mechanism was first demonstrated for normal conductors [26,27], then conjectured [13] and selfconsistently demonstrated [28-30] for the superconducting case. These results were corroborated recently by showing that the spiral order persists beyond the RKKY limit, and $k_{m}$ stays close to $k_{F}$, as long as $k_{F}$ is away from commensurate band fillings and the coupling strength $A$ between magnetic moments and electrons remains smaller than the electron bandwidth [31,32].

The locking of $k_{m}$ to $k_{F}$ has important consequences. The magnetic spiral forms a periodic superstructure that causes a part of the electrons to undergo a spin-selective Peierls transition [33] to a nonconducting spiral electron spin density wave, whereas the remaining conducting electron states become helical (spin filtered). The induced superconductivity then becomes of the topological $p$-wave type, and Majorana bound states appear at the two ends of the 1D wire. A system with such a spiral magnetic order is indeed equivalent [12-18,21,28-30,33-37] to the original proposals for topological superconductivity in nanowires $[7,8]$. Remarkably, by this mechanism, the topological superconducting phase emerges naturally as the ground state without any fine tuning. Although both the RKKY based and the nonperturbative approaches consistently predict the locking condition $k_{m} \sim k_{F}$, it must be stressed that the former results are based on the further condition of large magnetic and superconducting gap energies, whereas the latter apply only at zero temperature.

In this Rapid Communication, we provide a general analysis which incorporates entropy and thermal fluctuations in the nonzero temperature regime and we show that there exists a crossover to a magnetically ordered yet nontopological phase. Furthermore, we show that the spin-orbit interaction (SOI), which is genuinely present in such systems either intrinsically or through interface effects, causes a modification of the magnetic spiral but has no effect on the topological properties.

Heuristic considerations. The physical origin of the nontopological phase is illustrated in Fig. 2. At low temperatures $T$, the thermodynamic ground state is determined by the gain in electronic energy $E$ obtained by maintaining large magnetic $(J)$ and superconducting $\left(\Delta_{s}\right)$ gaps, and the system adjusts $k_{m}$ to $k_{F}$. As $T$ is raised, however, the ground state is dictated by the free energy $F=E-T S$, and the entropy $S$ can play a decisive role. Indeed, if $k_{m}$ is lowered or raised to the values as indicated in the left and right panels of Fig. 2, such that the chemical potential $\mu$ touches the bottom or top of a band, the induced superconducting gap closes (for $J>\Delta_{s}$ ) because the touched bands are fully spin polarized. The effective dispersion arising from the superconducting case becomes gapless, with a larger entropy than in the gapped case. As a result, if $T$ is large enough, typically $k_{B} T \lesssim \Delta_{S}$ (with $k_{B}$ the Boltzmann constant), the minimization of $F$ can be dominated by the enhancement of $S$, and the thermodynamic ground state corresponds to situations shown in Figs. 2(d) or 2(f), a topologically trivial yet magnetically ordered phase.

Not yet taken into account in this argument is the stability of this phase upon thermal fluctuations of the magnetic moments. As shown in Refs. [26-28], for both the ungapped and gapped cases, a mean-field description of the spin-wave fluctuations captures the correct value of the ordering temperature and 


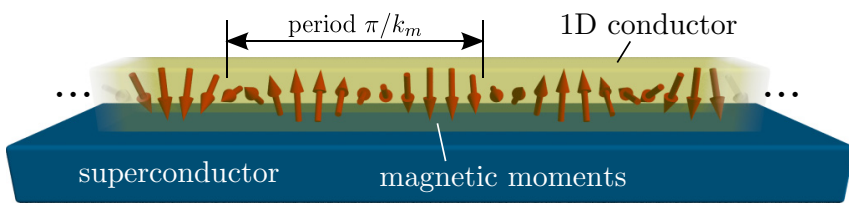

FIG. 1. (Color online) Zoom on a 1D conductor with embedded magnetic moments on top of a superconductor. The magnetic moments self-order in the form a spiral order with spatial period $\pi / k_{m}$.

$T^{*} \propto\left|\chi_{2 k_{m}}\right|$, with $\chi_{2 k_{m}}$ the transverse spin susceptibility at momentum $2 k_{m}$. Since $\left|\chi_{2 k_{m}}\right|$ increases for a gapless dispersion, closing the superconducting gap by moving $k_{m}$ away from $k_{F}$ causes furthermore an enhanced stability against thermal fluctuations, provided that the effect occurs at $T<T^{*}$. Obviously, the latter condition depends on the considered material as specified below. For practical implementations, the condition $k_{B} T^{*} \sim \Delta_{s}$ is a priori required for the topological self-tuning phase to be accessible at high enough temperatures, and for $T$ close to $T^{*}$ the nontopological ordered phase may indeed be favored. Yet we find that for semiconductor bands with an effective mass as in Fig. 2, the value of $T^{*}$ remains generally still too low, such that $S$ would dominate $E$ only at temperatures $T \gtrsim T^{*}$ where no order can persist anyway.

This situation changes drastically for tight binding systems such as shown in Fig. 3, which are the natural description for adatom chains. Due to the cosine nature of the dispersion, magnetic gaps appear at two points in the Brillouin zone, and $k_{m}$ can self-adjust such that the superconducting-magnetic gaps $\Delta^{*}$ at both points become equal and fulfill the condition
$\Delta^{*}<\left\{k_{B} T^{*}, \Delta_{s}\right\}$ [see Fig. 3(f)]. At $T \sim \Delta^{*}$ the effective doubling of thermally accessible states provides a doubling of the value of $S$. This is sufficient to push the transition to the nontopological to $T<T^{*}$ in precisely the systems that are most attractive for realizing a self-sustained topological phase. Furthermore, the equality of the two gaps $\Delta^{*}$ leads to $k_{m}=\pi / 2 a$ (with $a$ the lattice spacing), which corresponds to an antiferromagnetic arrangement of the magnetic moments if the latter are on the same lattice sites.

Quantitative analysis. For a quantitative investigation we consider a quantum wire with induced superconductivity and embedded magnetic moments, described by the Hamiltonian

$$
\begin{aligned}
H= & \sum_{k, \sigma}\left(\epsilon_{k}-\mu\right) c_{k, \sigma}^{\dagger} c_{k, \sigma}+\sum_{k, \sigma, \sigma^{\prime}}(\boldsymbol{\alpha} \cdot \boldsymbol{\sigma})_{\sigma, \sigma^{\prime}} k c_{k, \sigma}^{\dagger} c_{k, \sigma^{\prime}} \\
& +\sum_{k}\left(\Delta_{s} c_{k, \uparrow}^{\dagger} c_{-k, \downarrow}^{\dagger}+\text { H.c. }\right) \\
& +\sum_{k, q, \sigma, \sigma^{\prime}}\left(\mathbf{J}_{q} \cdot \boldsymbol{\sigma}\right)_{\sigma, \sigma^{\prime}} c_{k+q, \sigma}^{\dagger} c_{k, \sigma^{\prime}} .
\end{aligned}
$$

Here, $c_{k, \sigma}$ are the operators for electrons with spin $\sigma=\uparrow, \downarrow$ and dispersion relation $\epsilon_{k} \cdot \mu$ is the chemical potential, $\Delta_{s}$ the induced superconducting gap, $\sigma=\left(\sigma_{x}, \sigma_{y}, \sigma_{z}\right)$ the vector of Pauli matrices, and the vector $\boldsymbol{\alpha}$ the effective SOI in the system, arising from the sum of SOI contributions due to the internal structure of the wire or to interface effects with the substrate. The vectors $\mathbf{J}_{q}$ are the Fourier transforms of the chain of magnetic scatterers $\mathbf{J}_{i}=A \mathbf{I}_{i}$ coupling to the electron spin, where $\mathbf{I}_{i}$ are the magnetic moments and $A$ is the coupling strength. The $\mathbf{J}_{i}$ are placed at positions $r_{i}$ that can be irregular
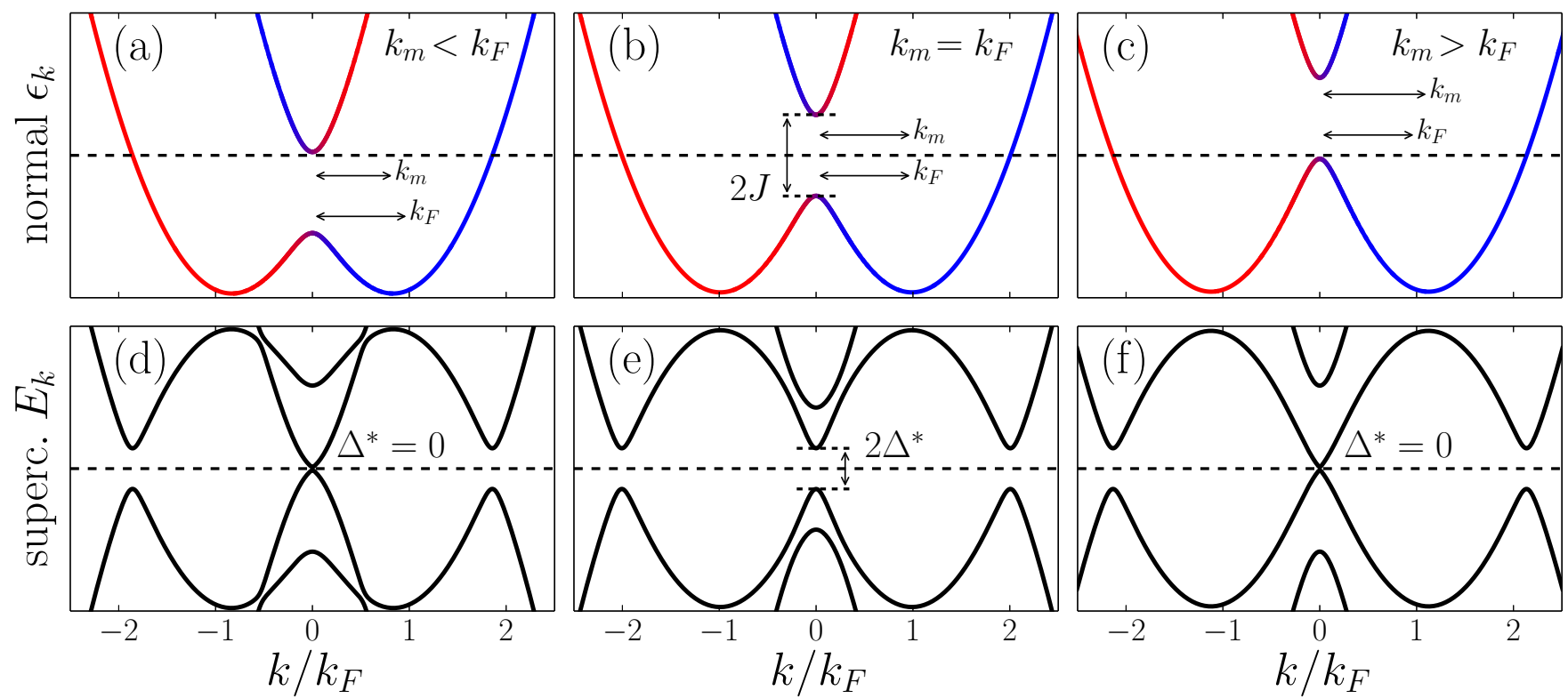

FIG. 2. (Color online) Example of dispersion relations in the transformed basis obtained by the spin-dependent shift of momenta $k \rightarrow$ $k+\sigma k_{m}$ for which the spiral effective magnetic field becomes ferromagnetic and the Zeeman-like gap $J$ opens at $k=0$ [33,38]. In the plots, the chemical potential $\mu$ remains constant, but $k_{m}$ varies. Top panels for the normal state $\left(\epsilon_{k}\right)$, with red and blue colors corresponding to opposite spin projections perpendicular to the spiral field. Bottom panels for the induced superconducting state $\left(E_{k}\right.$, with $\left.\Delta_{s}<J\right)$. For $k_{m}=k_{F}$, the chemical potential $\mu$ (dashed line) lies in the middle of the $J$ gap (b) and the superconducting system is fully gapped (e). For smaller or larger $k_{m}$, the gap lies at lower or higher energies and $\mu$ eventually touches the upper (a) or lower gap edge (c). At both touching points the superconducting gap closes [(d), (f)] and the state becomes nontopological. 

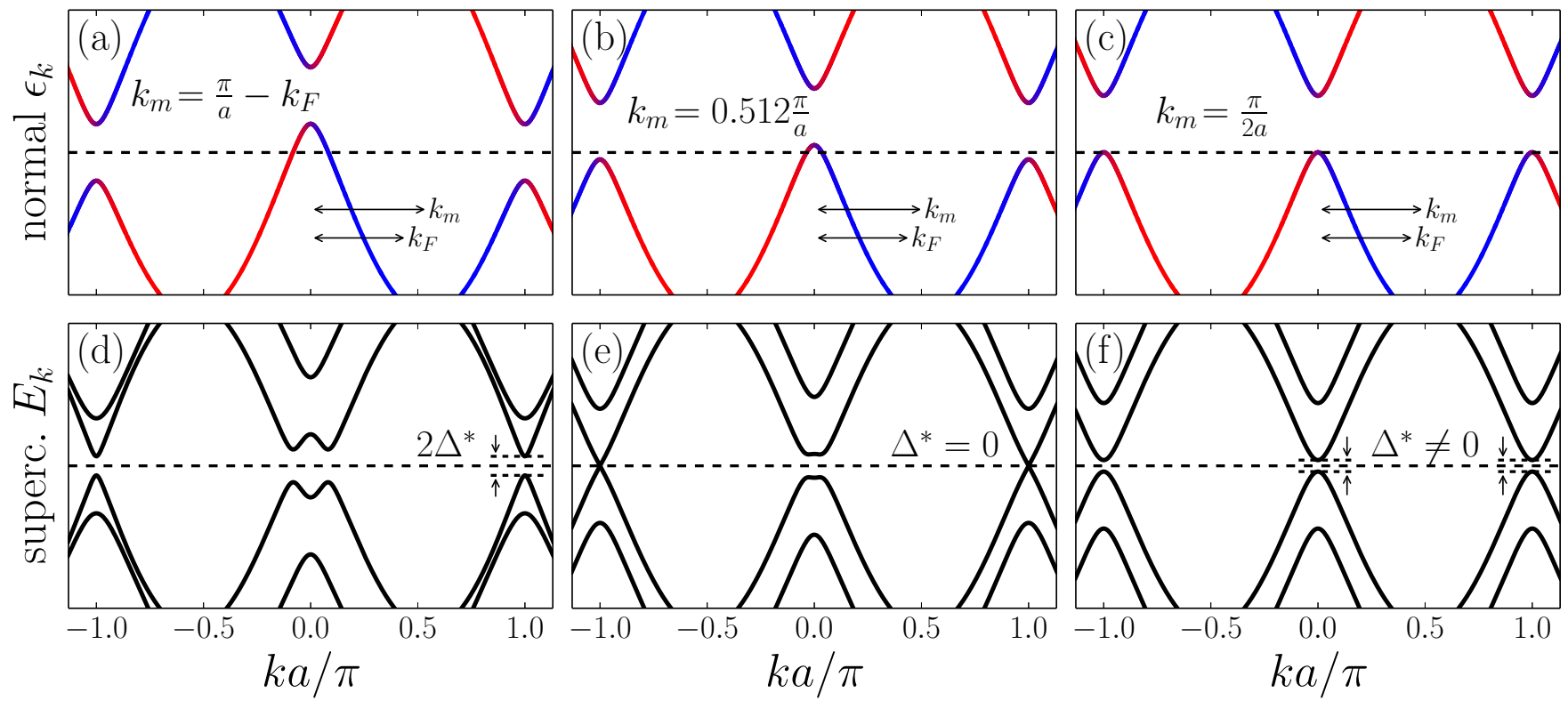

FIG. 3. (Color online) Dispersion relations as in Fig. 2 for the tight binding model associated with Eq. (1) and corresponding to Fig. 4. (a) and (d) represent the low-temperature topological phase, (b) and (e) the crossover to the nontopological phase where the gap $\Delta^{*}$ closes, and (c) and (f) the final high-temperature phase where $k_{m}=\pi / 2 a$ and the system is magnetically ordered yet nontopological.

but are sufficiently dense with respect to $2 \pi / k_{F}$ such that they can be considered as a continuum.

For $T=0$ and $\boldsymbol{\alpha}=\mathbf{0}$, the ground state energy is minimized if the vectors $\mathbf{J}_{i}$ are confined to an arbitrary twodimensional plane, spanned by orthogonal unit vectors $\left(\hat{\mathbf{e}}_{1}, \hat{\mathbf{e}}_{2}\right)$, in which they rotate as a spiral as a function of $r_{i}$, $\mathbf{J}_{i}=J\left[\cos \left(2 k_{m} r_{i}\right) \hat{\mathbf{e}}_{1}+\sin \left(2 k_{m} r_{i}\right) \hat{\mathbf{e}}_{2}\right][12,13,18,28-32,34,35]$. Choosing $\hat{\mathbf{x}}, \hat{\mathbf{y}}$ such that $(\hat{\mathbf{x}}, \hat{\mathbf{y}})=\left(\hat{\mathbf{e}}_{1}, \hat{\mathbf{e}}_{2}\right)$, the corresponding term in the Hamiltonian becomes $\sum_{k} J\left(c_{k+k_{m}, \uparrow}^{\dagger} c_{k-k_{m}, \downarrow}+\right.$ H.c. $)$. Letting $c_{k+\sigma k_{m}, \sigma} \rightarrow \tilde{c}_{k, \sigma}, \epsilon_{k} \rightarrow \epsilon_{k \mp \sigma k_{m}, \sigma}$ [33] produces a unitary transformation diagonalizing the Hamiltonian, in which $J$ forms a uniform ferromagnetic coupling along the spin- $x$ direction. For a parabolic dispersion $\epsilon_{k}=\hbar^{2} k^{2} / 2 m$ with the band mass $m$, we obtain Fig. 2 .

Since the SOI term is linear in $k$, a $\boldsymbol{\alpha} \neq \mathbf{0}$ produces a similar spin-dependent momentum shift. For a quadratic dispersion $\epsilon_{k}=\hbar^{2} k^{2} / 2 m$ with $m$ the band mass, and spin axes $\sigma_{\alpha}$ such that $\boldsymbol{\alpha} \cdot \boldsymbol{\sigma}=\alpha \sigma_{\alpha}$, the SOI can be absorbed

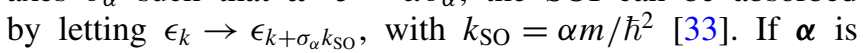
not perpendicular to the $\left(\hat{\mathbf{e}}_{1}, \hat{\mathbf{e}}_{2}\right)$ plane, the two shifts by $k_{\mathrm{SO}}$ and $k_{m}$ are not compatible, and the diagonalization of $H$ would generally mix all momenta. Such modifications of the long-ranged wave functions by the spiral order would cause an extensive energy cost which is not favored energetically. This can be avoided, however, by an alignment of the $\mathbf{J}_{i}$ spiral to the plane perpendicular to $\boldsymbol{\alpha}$. We thus define the spin directions such that $\boldsymbol{\alpha}=\alpha \hat{\mathbf{z}}$, and $\left(\hat{\mathbf{e}}_{1}, \hat{\mathbf{e}}_{2}\right)=(\hat{\mathbf{x}}, \hat{\mathbf{y}})$, for which $k_{\text {SO }}$ and $k_{m}$ are parallel and can be directly added. Remarkably, to maintain the optimal $k_{m}$ by minimizing the free energy, the spiral undergoes an adjustment of $k_{m}$ to a $k_{m}^{\prime}$ such that $k_{m}=k_{m}^{\prime}-k_{\text {SO }}$. While the "-" sign arises from the choice of spin axes, $k_{m}$ can have either sign, and so two spirals with opposite helicities and different periods, $k_{m}^{\prime}= \pm\left|k_{m}\right|+k_{\mathrm{SO}}$, are possible. Therefore, even a large SOI has no further influence than the pinning of the plane of the magnetic spiral together with the adjustment of $k_{m}^{\prime}$, provided that $2 \pi / k_{m}^{\prime}$ does not become smaller than the electron lattice spacing or the average spacing between the $\mathbf{J}_{i}$. As long as $k_{m}= \pm k_{F}$ (up to $J$-dependent corrections that can be included), a measurement of the period and plane of the magnetic spiral could therefore give a direct measurement of $\mathbf{k}_{\text {SO }} \propto \alpha$.

Due to the extensive energy cost, there are furthermore no conical deformations out of the spiral plane [18,39]. In a spin-Nambu matrix representation spanned by the vectors $\left(c_{k+k_{m}, \uparrow}^{\dagger}, c_{k-k_{m}, \downarrow}^{\dagger}, c_{-k+k_{m}, \uparrow}, c_{-k-k_{m}, \downarrow}\right)$, the Hamiltonian takes then the form

$$
H=\sum_{k>0}\left(\begin{array}{cccc}
\xi_{k-k_{m}} & J & 0 & \Delta_{s} \\
J & \xi_{k+k_{m}} & -\Delta_{s} & 0 \\
0 & -\Delta_{s}^{*} & -\xi_{-k-k_{m}} & -J \\
\Delta_{s}^{*} & 0 & -J & -\xi_{-k+k_{m}}
\end{array}\right)+E_{0}
$$

for $\xi_{k}=\epsilon_{k}-\mu, k_{m}=k_{m}^{\prime}-k_{\mathrm{SO}}, J_{q}=J \delta_{|q|,\left|2 k_{m}^{\prime}\right|}$, and the restriction of the summation to $k>0$ to avoid state overcounting. The energy offset is $E_{0}=\sum_{k>0}\left[\xi_{-k+k_{m}}+\xi_{-k-k_{m}}+2 J\right]$, and due to its $k_{m}$ dependence must be kept for a comparison of different $k_{m}$. The diagonalization of the matrix in Eq. (2), for $\xi_{-k}=\xi_{k}$, leads to the energies $E_{k}^{v, v^{\prime}}=v^{\prime} E_{k, v}$, for $v, v^{\prime}= \pm$, with $E_{k, \pm}^{2}=J^{2}+\Delta_{s}^{2}+$ $\xi_{+, k}^{2}+\xi_{-, k}^{2} \pm 2 \sqrt{\Delta_{s}^{2} J^{2}+\xi_{+, k}^{2}\left(J^{2}+\xi_{-, k}^{2}\right)}$ for $\xi_{ \pm, k}=\left(\xi_{k+k_{m}} \pm\right.$ $\left.\xi_{k-k_{m}}\right) / 2$. This leads to the ground state energy

$$
E=E_{0}+\sum_{k>0, v, v^{\prime}} E_{k}^{v, v^{\prime}} f_{k}^{v, v^{\prime}}
$$

and the entropy

$$
S=-k_{B} \sum_{k>0, v, v^{\prime}}\left[f_{k}^{v, v^{\prime}} \ln \left(f_{k}^{v, v^{\prime}}\right)+\left(1-f_{k}^{v, v^{\prime}}\right) \ln \left(1-f_{k}^{v, v^{\prime}}\right)\right],
$$


for $f_{k}^{v, v^{\prime}}=\left[1+\exp \left(E_{k}^{v, v^{\prime}} / k_{B} T\right)\right]^{-1}$ the Fermi function. Notice that the sums are restricted to $k>0$ to avoid overcounting. To proceed, we exclude the well-known scattering effects occurring at commensurate filling factors that can cause orderings different from spirals [31,32].

Minimizing the free energy $F=E-T S$ determines the ordering vector $k_{m}$. However, analyzing only $F$ is incomplete to assure the stability of the ordered phase since the longwavelength spin-wave fluctuations smooth any magnetization at finite $T$ for a system of finite size [26-30]. Taking this condition into account, it was demonstrated [27,28] that for any realistic system size, the mean-field result,

$$
k_{B} T^{*}=2 J^{2}\left|\chi_{2 k_{m}}\right| \sim \frac{J^{2} a^{\prime}}{\pi \hbar v_{F}} \ln \left(\frac{E_{F}}{\Gamma}\right),
$$

provides the ordering temperature $T^{*}$ for both the gapped and gapless cases, where $\chi_{2 k_{m}}$ is the static transverse spin susceptibility, expressed in terms of the Fermi energy $E_{F}=$ $\hbar v_{F} k_{F} / 2$, the Fermi velocity $v_{F}$, and the short distance cutoff $a^{\prime}$ (limited by lattice spacing $a$ or distance between the $\mathbf{J}_{i}$ ). If $\Delta^{*}$ is the gap, as indicated in Figs. 2 and 3, the energy $\Gamma$ is roughly set by $\max \left(\Delta^{*}, k_{B} T\right)$ and its value reflects the transition between the gapped $\left(\Delta^{*}>k_{B} T\right)$ and gapless regimes $\left(\Delta^{*}<k_{B} T\right)$. As a consequence, if $k_{m}$ departs from $k_{F}$ and $\Delta^{*}$ shrinks, the value of $T^{*}$ initially grows but then saturates at a self-consistent value where $\Gamma \sim k_{B} T^{*}$. Electron interactions further modify the logarithm in Eq. (5) to a temperature-dependent power law in the gapless case and can lead to a considerable further increase of $T^{*}[26,27]$. We note that Eq. (5) results from analyzing fluctuations arising from the RKKY interaction. While the RKKY limit is insufficient to characterize the ground state and $F$ needs to be used, it correctly encodes the fluctuations away from the ground state configuration for $J<a^{\prime} / \hbar v_{F}$.

Application to various systems. Since the transition to the nontopological phase occurs at $k_{B} T \lesssim \Delta_{s}$, materials with large $k_{B} T^{*} \sim \Delta_{s}$ shall be considered. With typical $\Delta_{s} \sim 0.1-1$ meV, most systems with nuclear magnetic moments have $k_{B} T^{*} \ll \Delta_{s}$, hence a guaranteed topological phase, albeit a low transition temperature $T^{*}$. For most 1D wire situations (including InAs [28]), we indeed find that $T^{*}$ remains too low to allow for a strong impact of the entropy, and the self-sustained topological phases remain stable.

Dense chains of magnetic adatoms on a superconducting substrate have a larger coupling constant $A$. If neighboring adatom orbitals hybridize, the chains become conductors of the type of Eq. (1). SOI effects are generally strong in such systems [23-25]. However, this causes here just a mere rearrangement of the magnetic helix $k_{m} \rightarrow k_{m}^{\prime}$. While systems such as in Refs. [23-25] likely depend much on the direct exchange interaction between neighboring moments, we focus here instead on the case where RKKY dominates over the latter (therefore our results do not a priori apply to Refs. [23-25]).

As demonstrated in Ref. [28], with $A \sim 0.5 \mathrm{meV}$ [40], we obtain $k_{B} T^{*}>\Delta_{s} \sim 1 \mathrm{meV}$ for the gapped phase. The requirement for such large $T^{*}$ is, as seen in Eq. (5), a large prefactor $a^{\prime} / \hbar v_{F}$, which means a rather small bandwidth or a renormalization of the Fermi velocity [41]. A tight binding model is therefore suitable, in which the factor $\hbar v_{F} / a^{\prime}$ is replaced by the hopping integral $t$, and the dispersion relation is $\epsilon_{k}=-2 t \cos (a k)$. Consequently, a minimum of $F$ at a shift $k_{m}$ has a particle-hole reversed minimum corresponding to the shift $\left(\pi / a-k_{m}\right)$. Away from half filling, $k_{F} \neq \pi / 2 a$, both minima are inequivalent, yet for $k_{F}$ not too far from $\pi / 2 a$, they can lie energetically close enough together such that further entropy can be gained by tuning $k_{m}$ through the topological boundary and pinning it to $k_{m}=\pi / 2 a$, an antiferromagnetic order at which the system is nontopological and has small gaps but with both minima contributing to $S$. In combination with a small bandwidth, a large $\Delta_{s}$, and $J \gtrsim \Delta_{s}$, the entropy can become large enough to dominate $F$. An example is given in Fig. 4, showing $F$ as a function of $k_{m}$ and $T$. The values
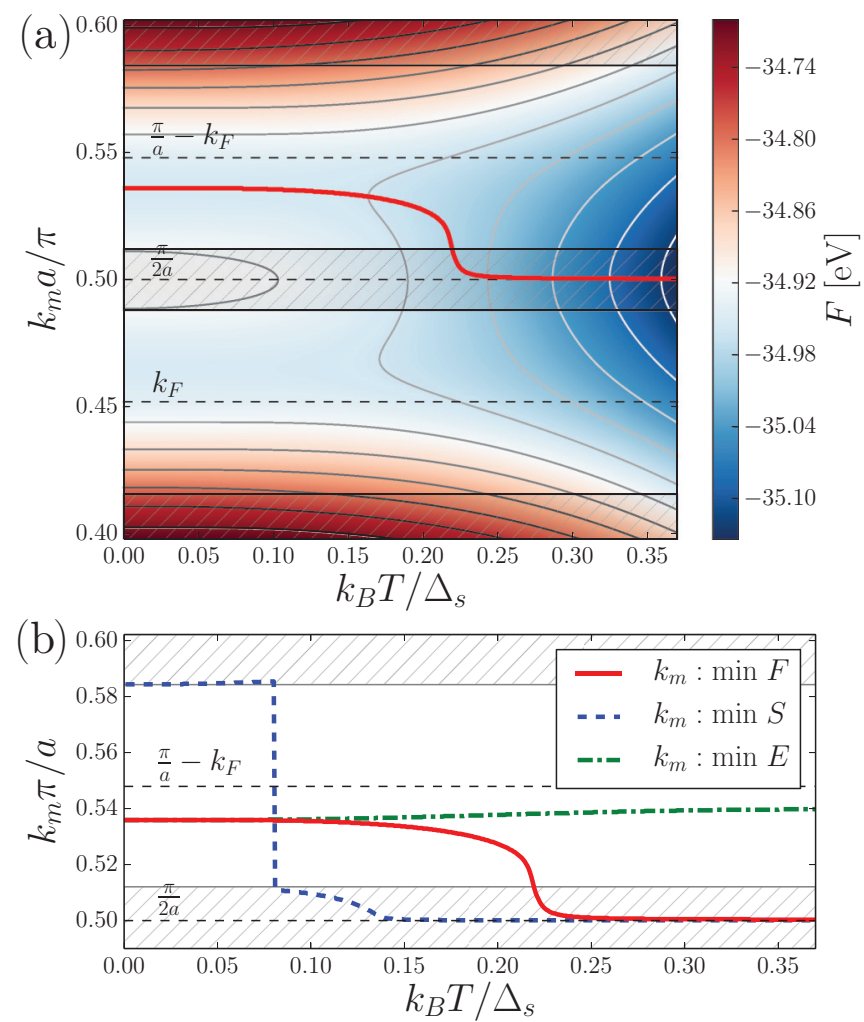

FIG. 4. (Color online) (a) Free energy $F$ for an adatom chain conductor of length $L=1 \mu \mathrm{m}$ as a function of total spiral momentum $k_{m}$ (including SOI contributions) and temperature $T$ [38]. Contour lines complement the color coding. The minima $k_{m}(T)$ are marked by the red line and correspond to the ground state configuration. The values $k_{F}, \pi / a-k_{F}$, and $\pi / 2 a$ are marked by horizontal dashed lines. The parameters for the tight binding model are $t=10 \mathrm{meV}, a=3 \AA$, $\Delta_{s}=2 \mathrm{meV}, J=3 \mathrm{meV}, L=1 \mu \mathrm{m}$, and $\mu=-0.3 t$ (7/20 filling). Stability of the order is ensured up to $k_{B} T^{*}=0.37 \Delta_{s}$ (at the right plot limits). The gap $\Delta^{*}$ closes at the phase boundary between the topological (clear) and nontopological (hatched) regions. The system becomes nontopological at $k_{B} T \approx 0.22 \Delta_{s}$ and $k_{m}$ then stabilizes at $\pi / 2 a$, corresponding to an antiferromagnetic order. (b) Values of $k_{m}$ minimizing the free energy $F$ [same as in (a)], the entropy $S$, and the energy $E$. While $E$ alone favors a topological phase, the entropy $S$ favors a gapless phase, but is at higher temperatures further enhanced by tuning $k_{m} \rightarrow \pi / a$, and eventually dominates the minimum of $F$ at $k_{B} T>0.22 \Delta_{s}$. For $k_{m}=\pi / 2 a$, the two identical gaps indicated in Fig. 3(f) are $\Delta^{*}=0.30 \Delta_{s}$. 
$k_{m}(T)$ minimizing $F(T)$ are indicated by the red line. At low temperatures $k_{m}$ lies near, but not on, $\left(\pi / a-k_{F}\right)$ since $J$ and $\Delta_{s}$ cause a significant band deformation due to the small bandwidth. At $k_{B} T \approx 0.22 \Delta_{s}$ we observe a crossing into the nontopological region (indicated by the hatching), well below $k_{B} T^{*} \approx 0.37 \Delta_{s}$, and the pinning to $k_{m}=\pi / 2 a$.

Conclusions. We have analyzed a 1D conductor with a spinorbit interaction coupled to a 1D chain of magnetic moments. Through a self-consistent analysis taking into account the full electronic free energy $F$ and the fluctuations about the ordered magnetic ground states, we have determined the stability of the topological superconducting phase at finite temperature. We showed that the spin-orbit interaction causes only a pinning of the plane of the magnetic spiral and an adjustment of its spatial period. Furthermore, in some situations especially met in systems of magnetic adatoms, we demonstrated that there is a significant temperature range, in which a magnetic order persists but the electronic state is nontopological.

Acknowledgments. We thank C. Carroll, J. Klinovaja, D. Loss, and D. Morr for helpful discussions. P.S. acknowledges support by the French Agence Nationale de la Recherche through the contract Mistral.
[1] M. Z. Hasan and C. L. Kane, Rev. Mod. Phys. 82, 3045 (2010).

[2] J. Alicea, Rep. Prog. Phys. 75, 076501 (2012).

[3] M. Leijnse and K. Flensberg, Semicond. Sci. Technol. 27, 124003 (2012).

[4] C. Nayak, S. H. Simon, A. Stern, M. Freedman, and S. Das Sarma, Rev. Mod. Phys. 80, 1083 (2008).

[5] J. K. Pachos, Introduction to Topological Quantum Computation (Cambridge University Press, Cambridge, U.K., 2012).

[6] L. Fu and C. L. Kane, Phys. Rev. Lett. 100, 096407 (2008).

[7] R. M. Lutchyn, J. D. Sau, and S. Das Sarma, Phys. Rev. Lett. 105, 077001 (2010).

[8] Y. Oreg, G. Refael, and F. von Oppen, Phys. Rev. Lett. 105, 177002 (2010).

[9] V. Mourik, K. Zuo, S. M. Frolov, S. R. Plissard, E. P. A. M. Bakkers, and L. P. Kouwenhoven, Science 336, 1003 (2012).

[10] A. Das, Y. Ronen, Y. Most, Y. Oreg, M. Heiblum, and H. Shtrikman, Nano Lett. 8, 887 (2012).

[11] M. T. Deng, C. L. Yu, G. Y. Huang, M. Larsson, P. Caroff, and H. Q. Xu, Nano Lett. 12, 6414 (2012).

[12] T.-P. Choy, J. M. Edge, A. R. Akhmerov, and C. W. J. Beenakker, Phys. Rev. B 84, 195442 (2011).

[13] S. Nadj-Perge, I. K. Drozdov, B. A. Bernevig, and A. Yazdani, Phys. Rev. B 88, 020407(R) (2013).

[14] F. Pientka, L. I. Glazman, and F. von Oppen, Phys. Rev. B 88, 155420 (2013).

[15] F. Pientka, L. I. Glazman, and F. von Oppen, Phys. Rev. B 89, 180505(R) (2014).

[16] K. Pöyhönen, A. Westström, J. Röntynen, and T. Ojanen, Phys. Rev. B 89, 115109 (2014).

[17] J. Röntynen and T. Ojanen, Phys. Rev. B 90, 180503 (2014).

[18] Y. Kim, M. Cheng, B. Bauer, R. M. Lutchyn, and S. Das Sarma, Phys. Rev. B 90, 060401(R) (2014).

[19] A. Heimes, P. Kotetes, and G. Schön, Phys. Rev. B 90, 060507(R) (2014).

[20] P. M. R. Brydon, S. Das Sarma, H.-Y. Hui, and J. D. Sau, Phys. Rev. B 91, 064505 (2015).

[21] K. Pöyhönen, A. Westström, and T. Ojanen, arXiv:1509.05223.

[22] M. Schecter, K. Flensberg, M. H. Christensen, B. M. Andersen, and J. Paaske, arXiv:1509.07399.

[23] S. Nadj-Perge, I. K. Drozdov, J. Li, H. Chen, S. Jeon, J. Seo, A. H. MacDonald, B. A. Bernevig, and A. Yazdani, Science 346, 602 (2014).
[24] R. Pawlak, M. Kisiel, J. Klinovaja, T. Meier, S. Kawai, T. Glatzel, D. Loss, and E. Meyer, arXiv:1505.06078.

[25] M. Ruby, F. Pientka, Y. Peng, F. von Oppen, B. W. Heinrich, and K. J. Franke, Phys. Rev. Lett. 115, 197204 (2015).

[26] B. Braunecker, P. Simon, and D. Loss, Phys. Rev. Lett. 102, 116403 (2009).

[27] B. Braunecker, P. Simon, and D. Loss, Phys. Rev. B 80, 165119 (2009).

[28] B. Braunecker and P. Simon, Phys. Rev. Lett. 111, 147202 (2013).

[29] J. Klinovaja, P. Stano, A. Yazdani, and D. Loss, Phys. Rev. Lett. 111, 186805 (2013).

[30] M. M. Vazifeh and M. Franz, Phys. Rev. Lett. 111, 206802 (2013).

[31] M. Schecter, M. S. Rudner, and K. Flensberg, Phys. Rev. Lett. 114, 247205 (2015).

[32] W. Hu, R. T. Scalettar, and R. R. P. Singh, Phys. Rev. B 92, 115133 (2015).

[33] B. Braunecker, G. I. Japaridze, J. Klinovaja, and D. Loss, Phys. Rev. B 82, 045127 (2010).

[34] M. Kjaergaard, K. Wölms, and K. Flensberg, Phys. Rev. B 85, 020503(R) (2012).

[35] I. Martin and A. Morpurgo, Phys. Rev. B 85, 144505 (2012).

[36] J. Klinovaja, P. Stano, and D. Loss, Phys. Rev. Lett. 109, 236801 (2012).

[37] C.-H. Hsu, P. Stano, J. Klinovaja, and D. Loss, arXiv: 1509.01685 .

[38] For a magnetic spiral, the shift $k \rightarrow k \mp \sigma k_{m}$ diagonalizes the Hamiltonian without changing the energy, and hence the results for different $k_{m}$ are directly comparable. The same type of transformation also diagonalizes the SOI but adds an energy offset $\alpha^{2} m / 2$. However, since the SOI is fixed for a given system, this offset is the same for all further magnetic spirals and has no effect on the discussion.

[39] I. Reis, D. J. J. Marchand, and M. Franz, Phys. Rev. B 90, 085124 (2014).

[40] M. Menzel, Y. Mokrousov, R. Wieser, J. E. Bickel, E. Vedmedenko, S. Blügel, S. Heinze, K. von Bergmann, A. Kubetzka, and R. Wiesendanger, Phys. Rev. Lett. 108, 197204 (2012).

[41] M. Ruby, F. Pientka, Y. Peng, F. von Oppen, B. W. Heinrich, and K. J. Franke, Phys. Rev. Lett. 115, 087001 (2015). 